J3eA, Journal sur l'enseignement des sciences et technologies de l'information et des systèmes,

Volume 3, Hors-Série 1, 9 (2004)

DOI : http://dx.doi.org/10.1051/bib-j3ea:2004609

(C) EDP Sciences, 2004

Robotique et compensation du handicap locomoteur chez les personnes âgées : déambulateurs intelligents

P. Rumeau ${ }^{1,2,4}$, S. Moulias ${ }^{4}$, V. Pasqui ${ }^{3}$, F. Steenkeste ${ }^{2}$, B. Vellas ${ }^{1}$ et P. Bidaud ${ }^{3}$

${ }^{1}$ CHU Toulouse, Service de Médecine Interne et Gérontologie Clinique, France

${ }^{2}$ INSERM U558, Toulouse, Fance

${ }^{3}$ Laboratoire de Robotique de Paris, Paris VI, France

${ }^{4}$ Cellule Gérontechnologies, CHU Charles Foix, Ivry-sur-Seine, France 


\title{
Robotique et compensation du handicap locomoteur chez les personnes âgées : déambulateurs intelligents
}

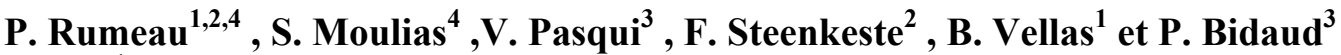 \\ ${ }^{1} \mathrm{CHU}$ Toulouse, Service de Médecine Interne et Gérontologie Clinique \\ ${ }^{2}$ Inserm U558, Toulouse \\ ${ }^{3}$ Laboratoire de Robotique de Paris, Paris VI \\ ${ }^{4}$ Cellule Gérontechnologies, CHU Charles Foix, Ivry sur Seine
}

Dans les pays de l'Union Européenne on estime que plus de $10 \%$ de la population présente un handicap, soit de l'ordre de 40 millions de citoyens. 4 personnes de plus de 75 ans sur 10 ont une limitation fonctionnelle sévère [5]. Prés de $8 \%$ la population française à plus de 75 ans [4] et une personne sur trois de cet âge chute au moins une fois dans l'année [1,6]. Plus de la moitié des pensionnaires de maison de retraite sont considérés comme chuteurs [5]. Ce trouble de la marche est multifactoriel par nature.

Une marche efficace doit répondre à plusieurs critères : être stable, contrôlée et économique du point de vue énergétique. Ces critères ne sont en pratique hautement intriqués ; une marche avec un comportement de déambulation sera plus coûteuse en fatigue et une chute aura une probabilité accrue de survenir. Vieillissement et pathologie vont agir sur ces trois critères.

Avec le vieillissement neurologique les informations afférentes sur le corps (somesthésie) et son environnement (exteroception) deviennent erronées à $30 \%$ [7]. L'individus est obligé de "faire attention », la marche d'automatique devient de plus en plus volontaire. La marge d'erreur des afférences est compensée par des mesures répétées, une étude évolutive et un moyennage. Le mécanisme de commande passe d'un système en proaction chez le sujet jeune à un système en rétroaction chez le sujet âgée. Le patern moteur est redéfini en permanence au prix d'un coût accru en travail musculaire et donc d'une plus forte dépense énergétique. Cette théorie reflète bien l'augmentation progressive du risque de chute passés 60 ans. De même, nous comprenons que la vitesse confortable de marche va progressivement diminuer : $1,45 \mathrm{~m} / \mathrm{s}$ chez un adulte de 20 ans, $1,23 \mathrm{~m} / \mathrm{s}$ chez un homme de 60 ans, $1,18 \mathrm{~m} / \mathrm{s}$ chez un homme de 80 ans [7]. Le vieillissement va également toucher les fonctions sensorielles. La vision et l'ouïe ont un rôle majeur dans la détection de modifications soudaines de l'environnement. La vision est touchée systématiquement par la presbytie : le cristallin devient moins plastique et perd des capacités d'accommodation, la vision de prés devient déficitaire, les lunettes à double foyer ont une effet déformant qui peut causer des chutes notamment dans les escaliers. La dégénérescence maculaire liée à l'âge, nécessitant un usage plus important de la rétine périphérique peut survenir, elle peut empêcher la lecture de panneaux de signalisation.

Aux effets du vieillissement s'ajoutent ceux de la pathologie qu'elle soit due à l'accumulation de déficiences avec le temps ou qu'il s'agisse de maladies spécifiques à l'age. Les pathologies locomotrices extrêmement fréquentes (arthrose, séquelles de fracture du col du fémur ou d'hémiplégie même incomplète) vont souvent nécessiter l'utilisation d'une aide technique pour stabiliser la marche: canne, déambulateur. Les pathologies neuro-cognitives, au premier rang desquelles la maladie d'Alzheimer, sont une cause d'altération de la marche qui atteignent un niveau épidémique avec l'augmentation de l'espérance de vie. Dés le début d'une maladie d'Alzheimer les patients peuvent se perdre du fait d'une désorientation temporelle et spatiale. Par la suite les effets cognitifs de la maladie s'accompagnent d'un effet direct sur la fragilité des automatismes de la marche qui peuvent être perdus en quelques jours d'alitement pour une pathologie intercurrente. Cette pathologie induit également de comportement de déambulation qui peuvent amener le patient à un épuisement physique et à la perte de la capacité à marcher. Enfin dans les formes évoluées le patient va perdre la marche par apragmatisme ; il n'a même plus l'idée de vouloir se déplacer et le schéma moteur s'efface par inutilisation.

Le caractère plurifactoriel du handicap à la marche chez les personnes âgées appelle une réponse multiple. L'aide technique idéale devrait à la fois fournir un soutien mécanique et une aide à la "commande » capable de s'adapter à l'environnement extérieur et aux incapacités de l'utilisateur. Ce cahier des charge correspond pratiquement à la définition d'une aide robotique. Le terme de robotique, potentiellement choquant du fait de l'inconscient collectif (Golem, remplacement de l'homme par la machine au XIXéme siècles, Frankenstein...) sera remplacé par le terme d'intelligent (intelligent ou smart en anglais) terme plus neutre au vécu positif.

Plusieurs démbulateurs intelligents ont été développés depuis dix ans. Guido ${ }^{\circledR}$ développé par $\mathrm{G}$. Lacey est le système actuellement le plus aboutit et le seul commercialisé. Il s'agit d'un système développé comme aide technique spécifique pour les personnes associant une déficience visuelle et motrice. Si une personne aveugle devient instable à la marche elle ne peut plus utiliser une canne ou son chien; une aide humaine devient nécessaire pour la guider en plus d'une aide technique à la marche. La personne est donc dépendante de la disponibilité d'un guide humain. Guido ${ }^{\circledR}$ se présente comme un déambulateur de type «rolator». Guido ${ }^{\circledR}$ est motorisé par la poussée de l'utilisateur. Il est fait pour s'utiliser en terrain plat et plutôt en intérieur (roues, contraintes de stabilité, utilisateurs potentiels). Il 
localise les obstacles frontaux par laser et latéraux par sonar, des capteurs de position contrôlent où il a été déplacé. Une synthèse vocale permet d'avertir l'utilisateur des obstacles et des possibilités de navigation (croisements notamment). L'asservissement de la direction à la détection d'obstacle peut être débrayé si l'utilisateur le souhaite. Il a un bouton permettant à l'utilisateur de le tourner sur son propre axe pour les lieux étroits. Guido ${ }^{\circledR}$ a vu son usage validé par des tests d'utilisation dans une maison de retraite de Dublin. L'obstacle majeur à sa diffusion auprès des utilisateurs potentiel est son prix (dans les 12.500 euros, plus qu'une petite voiture !!!).

D'autres systèmes sont à l'état de prototype. CareO-Bot du Fraunhofer Institute de Stuttgart (98) est un déambulateur motorisé avec un système de navigation et d'évitement des obstacles et un bras de manipulation, il est destiné à des gens utilisant déjà un déambulateur. Le PAMM de Dubowsky (MIT 2000) est un déambulateur motorisé associant au système d'évitement d'obstacle un système de navigation pour les personne désorientées. Cette navigation fonctionne par reconnaissance optique d'un code visuel binaire peint au plafond du lieu de vie.

D'autres déambulateurs intelligents sont au stade de projet. Le projet MONIMAD est un projet soutenu par le Réseau national Technologie et Santé. Il s'agit d'un déambulateur qui sur des fonctionnalités de base communes (évitement d'obstacle, surveillance du pouls, aide pour se lever et s'asseoir) ajoutera des fonctionnalités modulaires au besoin (détecteur de chute, mesure de la puissance à développé par l'utilisateur et donc du travail cardiaque...).

Il est possible de dire que pour être qualifiée de déambulateur intelligent et être acceptable en pratique médicale une aide technique doit répondre à un cahier des charges minimum :

- Absence de dangerosité pour l'utilisateur et son entourage

- Utilisation en intérieur

- Aide à la stabilité à la marche et aux transferts

- Support à la marche

- Aide à l'évitement d'obstacles

- Simplicité d'utilisation

- Maniabilité (tourner sur place notamment)

- Robustesse

- Pas de technologie apparente

- Compatibilité avec les normes de sécurité

- Coût en rapport avec les possibilités financières des utilisateurs ou avec leurs possibilité de soutien social (solidarité).

La robotique par sa définition répond aux exigences de systèmes de compensation du handicap multifactoriel propre aux personnes âgées fragiles. Des systèmes pertinents sont déjà disponibles mais se heurtent à une problématique financière. La poursuite de l'effort de recherche et de coopération entre les mondes de la recherche, de l'industrie et de la gérontologie médicale devraient permettre de développer des déambulateurs intelligents participant à l'accroissement de l'autonomie, la liberté de choix et la qualité de la vie des personnes âgées handicapées.

\section{BIBLIOGRAPHIE}

[1] Campbell AJ, Borrie MJ, Spears GF. Risk factors for falls in a community-based prospective study of people 70 years and older. J Gerontol 1989 ; 44(4) : M112-M117.

[2] Jensen J, Lundin-Olsson L, Nyberg L, Gustafson Y. Fall and injury prevention in older people living in residential care facilities. Ann Intern Med 2002; 136 : 733-741.

[3] Lauritzen JB, Petersen NM, Lund B. Effect of external hip protectors on hip fractures. Lancet 1993 ; 341 : 11-13.

[4] Recensement de la population 1999 - Exploitation principale.

http://WWW.Recensement.insee.fr.

[5] Sandhu Jim, Carruthers Start, Delaney Lesa. Demography trends and issues. Proceedings of the fifth COST 219 conference, Tregastel, 7 et 8 juin 1994.

[6] Tinetti ME, Speechly M, Ginter SF. Risk factors for falls among elderly persons living in the community. $\mathrm{N}$ Engl J Med $1988 ; 319$ : 1701-1707.

[7] Vieillissement et psychomotricité. Sous la direction de JM Albaret et E Aubert, Solal, Marseille 2001. 